\title{
Lipid resuscitation: development in basic research and application to clinical practice
}

\author{
Yutaka Oda
}

Received: 30 June 2013 / Accepted: 23 July 2013/Published online: 13 August 2013

(C) Japanese Society of Anesthesiologists 2013

Keywords Local anesthetics - Lipid emulsion · Resuscitation · Pediatric

\section{Background}

It has been nearly 15 years since the effectiveness of intravenous lipid emulsion in resuscitation from bupivacaine-induced cardiac arrest was reported by Weinberg et al. [1]. Despite the outstanding novelty and originality of this finding, it was not until a 2006 case report of a patient resuscitated with lipid emulsion after bupivacaine-induced refractory dysrhythmia and cardiac arrest that its clinical effectiveness was acknowledged [2]. This event was followed by a large number of case reports that showed the effectiveness of lipid emulsion for treating the central nervous system as well as cardiac toxicity induced by various kinds of local anesthetics [3-8]. Treatment with intravenous lipid emulsion has been incorporated into practice advisories of the Association of Anesthesiologists of Great Britain and Ireland, ${ }^{1}$ the American Society of Regional Anesthesia and Pain Medicine [9], and endorsed by the American Heart Association Advanced Cardiac Life Support (ACLS) guidelines [10] (Table 1).

In response to widespread interest, numerous studies have been undertaken using various animal models [8].

Y. Oda $(\bowtie)$

Department of Anesthesiology, Osaka City General Hospital

and Children's Hospital 2-2-43, Miyakojima-hondori,

Miyakojima-ku, Osaka 534-0021, Japan

e-mail: odayou@msic.med.osaka-cu.ac.jp
Notably, most of the animal experiments have been focused on examining the effect of lipid on local anesthetic-induced cardiac toxicity, not central nervous system toxicity, probably because of the primary concern of resuscitation and the entirely different animal models required for studying these two types of toxicity. Animals under mechanical ventilation with oxygen are needed to examine the effect of lipid emulsion on cardiac toxicity of local anesthetics while eliminating the influence of hypoxia induced by sedation and convulsions preceding circulatory collapse [11-14]. In contrast, awake, spontaneously breathing animals are needed to observe its effect on the central nervous system toxicity [15]. Randomized clinical trials are no longer ethically defensible for studying local anesthetic toxicity. Moreover, clinical data from healthy volunteers given nontoxic doses of bupivacaine would not precisely reflect the condition at the onset of the toxic effects [16].

\section{Mechanism}

The primary objective of these animal studies was to elucidate the mechanisms of reversal of the toxic effects of local anesthetics, apart from merely examining the effect of lipid emulsion on their toxicity [11-15]. Two distinct effects, partitioning and enhanced metabolism, have been suggested as possible mechanisms of the antidote effect of lipid emulsion, although the effects are complicated and cannot be explained simply [8].

Regarding partitioning, the early evidence favored an especially important role for the so-called "lipid sink," the creation of an intravascular lipid compartment that

\footnotetext{
${ }^{1}$ http://www.aagbi.org/publications/publications-guidelines/.
} 
Table 1 Recommended procedure for lipid resuscitation for local anesthetic overdose

1. Call for help

2. Ventilate with pure oxygen: suppress seizure with benzodiazepines

3. Basic and Advanced Cardiac Life Support (ACLS)

Avoid vasopressin, calcium channel blockers, beta blockers, or local anesthetics

Reduce individual adrenaline doses ${ }^{\mathrm{a}}$

4. Infusion of $20 \%$ lipid emulsion

Bolus $1.5 \mathrm{ml} / \mathrm{kg}$, followed by continuous infusion $0.25 \mathrm{ml} / \mathrm{kg} / \mathrm{min}$

Repeat bolus infusion once or twice

Infusion rate should be increased to $0.5 \mathrm{ml} / \mathrm{kg} / \mathrm{min}$ if blood pressure remains low

Continue infusion for at least 10 min after attaining circulatory stability

Recommended upper limit: approximately $10 \mathrm{ml} / \mathrm{kg}$ over the first $30 \mathrm{~min}^{\mathrm{b}}$

${ }^{a}$ Individual doses $<1 \mu \mathrm{g} / \mathrm{kg}$ is recommended by the American Society of Regional Anesthesia and Pain Medicine [9]

b Exceeding this limit is allowed in patients with persistent dysrhythmia or hypotension

sequesters bupivacaine from the plasma phase and allows its effective plasma concentration to decrease; this is the most plausible explanation for its effectiveness on both the central nervous system and cardiac toxicity of local anesthetics. The lipid sink hypothesis is also supported by the findings that the brain does not metabolize fatty acids as an energy source and that lipid emulsion reverses the toxicity caused by an array of agents lacking a common mechanism, site of action, chemical structure, or clinical effect [8, 17].

A long time after Weinberg's first report [1] showing a decrease of free bupivacaine in plasma after mixing with lipid emulsion, French et al. [18] revealed that adding lipid emulsion to human serum decreased the drug concentration in the aqueous layer in a manner dependent on the aqueous/ lipid partition coefficient. Lipid emulsion also substantially reduced methemoglobin production caused by the three most lipid soluble drugs tested, but not by the other less lipid soluble ones in vitro [19]. Recently, enhanced decrease of the concentration of bupivacaine in multiple organs including the brain and heart by lipid emulsion was shown in rats and in a physiologically based pharmacokinetic model $[13,20]$. Taken together, these studies support the role of partitioning in lipid resuscitation in treating bupivacaine toxicity and overdose of other lipophilic drugs. Lipid flux is also a possible explanation of the effect of lipid emulsion from the aspect of metabolism [21]. Lipid emulsion offsets the potent inhibition of fatty acid metabolism by bupivacaine and improves energy supply to cardiac muscles $[8,12]$.
Use of adrenaline in resuscitation for local anesthetic overdose

Adrenaline has been extensively used for resuscitation of patients with bradycardia, pulseless ventricular tachycardia, and asystole, and its use is also recommended in the ACLS protocol. On the other hand, local anestheticinduced dysrhythmia and asystole are resistant to treatment with adrenaline [2, 3, 5]. Notably, recovery of cardiac function after starting infusion of lipid emulsion without adrenaline has also been reported [4]. Moreover, a single dose of adrenaline at $\geq 10 \mu \mathrm{g} / \mathrm{kg}$ significantly impaired the efficacy of lipid resuscitation despite a rapid increase of rate pressure product early in the resuscitation [11]. Adding adrenaline to lipid emulsion induced ventricular tachycardia and did not improve recovery time from bupivacaineinduced asystole in an isolated rat heart [22]. Lipid emulsion was also superior to vasopressin for resuscitation of bupivacaine-induced cardiac arrest in a rat model [14]. These findings could have probably resulted from the different causes of cardiovascular collapse in local anesthetic systemic toxicity compared with other more common causes of cardiac arrest, such as myocardial ischemia. Removal of local anesthetics from cardiac tissue is most effective and crucially important for treating local anesthetic-induced toxicity. An increase of peripheral vascular resistance with potent vasopressors may impair cardiac output and impede resuscitation. According to the checklist for treatment of local anesthetic systemic toxicity by the American Society of Regional Anesthesia and Pain Medicine [9], individual doses of adrenaline should be less than $1 \mu \mathrm{g} / \mathrm{kg}$.

\section{Use of lipid emulsion in pediatric patients}

Lipid emulsion has been licensed for pediatric use as parenteral nutrition for more than 20 years and has an excellent safety profile, albeit at infused doses much lower than those recommended for resuscitation from drug toxicity. Its effectiveness in treating toxic effects of various agents including local anesthetics and psychomotor agents in children as well as in adults have been reported [17]. Although a paucity of data prevents determination of the adequate dose of lipid emulsion in children, $\leq 10 \mathrm{ml} / \mathrm{kg}$ has been used for resuscitation in most cases, which is comparable with that reported in adults $[5,8,17,23]$. Further studies on the pharmacokinetics and toxicity of local anesthetics in children will provide new information on the use of lipid emulsion.

The recommended maximum doses of lipid emulsion are 10 and $12 \mathrm{ml} / \mathrm{kg}$, according to the American Society of Regional Anesthesia and Pain Medicine [9] and the 
Association of Anesthesiologists of Great Britain and Ireland, respectively. These values are probably based on previous clinical reports showing that cardiovascular failure was resuscitated by lipid emulsion at $\leq 10 \mathrm{ml} / \mathrm{kg}$ in most cases $[8,17]$. However, these values should not be regarded as absolute upper limits, and infusion of lipid emulsion should be continued in the presence of refractory dysrhythmias and circulatory collapse, as cases of both adults and children requiring lipid emulsion at $>15 \mathrm{ml} / \mathrm{kg}$ for successful complication-free resuscitation from drug-induced cardiac arrest have been reported $[2,24]$.

\section{Adverse effects}

Only a few adverse effects have been reported among a large number of cases of resuscitation by lipid emulsion. Levine et al. [23] reported hypertriglyceridemia and pancreatitis in a 13-year-old girl after resuscitation from cardiovascular collapse induced by amitriptyline, with $20 \%$ lipid emulsion given as a $1.5 \mathrm{ml} / \mathrm{kg}$ bolus and $0.25 \mathrm{ml} / \mathrm{kg} / \mathrm{min}$ infusion for $30 \mathrm{~min}$. A remarkable increase of serum triglyceride and lipase levels was detected after resuscitation, resulting in a prolonged hospital stay. Sirianni et al. [25] reported acute lung injury, requiring mechanical ventilation for more than 2 weeks in a 17-year-old girl who was resuscitated from cardiovascular collapse, after intentional ingestion of an overdose of bupropion and lamotrigine, by a single $100-\mathrm{ml}$ bolus of $20 \%$ lipid emulsion, although causal relationship between prolonged hypotension and the lung injury cannot be completely ruled out. Although reported so far only in animal experiments, generalized mottling of the skin following lipid infusion, consistent with complement activation-related pseudoallergy, has also been seen in pigs [26].

In conclusion, intravenous lipid emulsion is effective for treating toxicity caused by various lipophilic agents including local anesthetics. Although the detailed mechanism is not clear, a lipid sink appears to play an important role in its effectiveness as an antidote.

\section{References}

1. Weinberg GL, VadeBoncouer T, Ramaraju GA, Garcia-Amaro MF, Cwik MJ. Pretreatment or resuscitation with a lipid infusion shifts the dose-response to bupivacaine-induced asystole in rats. Anesthesiology. 1998;88:1071-5.

2. Rosenblatt MA, Abel M, Fischer GW, Itzkovich CJ, Eisenkraft JB. Successful use of a $20 \%$ lipid emulsion to resuscitate a patient after a presumed bupivacaine-related cardiac arrest. Anesthesiology. 2006;105:217-8.
3. Litz RJ, Popp M, Stehr SN, Koch T. Successful resuscitation of a patient with ropivacaine-induced asystole after axillary plexus block using lipid infusion. Anaesthesia. 2006;61:800-1.

4. Foxall G, McCahon R, Lamb J, Hardman JG, Bedforth NM. Levobupivacaine-induced seizures and cardiovascular collapse treated with Intralipid. Anaesthesia. 2007;62:516-8.

5. Shah S, Gopalakrishnan S, Apuya J, Martin T. Use of Intralipid in an infant with impending cardiovascular collapse due to local anesthetic toxicity. J Anesth. 2009;23:439-41.

6. Mizutani K, Oda Y, Sato H. Successful treatment of ropivacaineinduced central nervous system toxicity by use of lipid emulsion: effect on total and unbound plasma fractions. J Anesth. 2011;25: $442-5$.

7. Nguyen VH, White JL. Further support for the early administration of lipid emulsion in the treatment of ropivacaine-induced central nervous system toxicity. J Anesth. 2012;26:479-80.

8. Weinberg GL. Lipid emulsion infusion: resuscitation for local anesthetic and other drug overdose. Anesthesiology. 2012;117: $180-7$.

9. Neal JM, Mulroy MF, Weinberg GL. American Society of Regional Anesthesia and Pain Medicine checklist for managing local anesthetic systemic toxicity: 2012 version. Reg Anesth Pain Med. 2012;37:16-8.

10. Vanden Hoek TL, Morrison LJ, Shuster M, Donnino M, Sinz E, Lavonas EJ, Jeejeebhoy FM, Gabrielli A. Part 12: cardiac arrest in special situations: 2010 American Heart Association Guidelines for Cardiopulmonary Resuscitation and Emergency Cardiovascular Care. Circulation. 2010;122:S829-61.

11. Hiller DB, Gregorio GD, Ripper R, Kelly K, Massad M, Edelman L, Edelman G, Feinstein DL, Weinberg GL. Epinephrine impairs lipid resuscitation from bupivacaine overdose: a threshold effect. Anesthesiology 2009;111:498-505.

12. Partownavid P, Umar S, Li J, Rahman S, Eghbali M. Fatty-acid oxidation and calcium homeostasis are involved in the rescue of bupivacaine-induced cardiotoxicity by lipid emulsion in rats. Crit Care Med. 2012;40:2431-7.

13. Shi K, Xia Y, Wang Q, Wu Y, Dong X, Chen C, Tang W, Zhang Y, Luo M, Wang X, Papadimos TJ, Xu X. The effect of lipid emulsion on pharmacokinetics and tissue distribution of bupivacaine in rats. Anesth Analg. 2013;116:804-9.

14. Di Gregorio G, Schwartz D, Ripper R, Kelly K, Feinstein DL, Minshall RD, Massad M, Ori C, Weinberg GL. Lipid emulsion is superior to vasopressin in a rodent model of resuscitation from toxin-induced cardiac arrest. Crit Care Med. 2009;37:993-9.

15. Oda Y, Ikeda Y. Effect of lipid emulsion on the central nervous system and cardiac toxicity of bupivacaine and levobupivacaine in awake rats. J Anesth 2013 Mar 14 (Epub ahead of print).

16. Litonius E, Tarkkila P, Neuvonen PJ, Rosenberg PH. Effect of intravenous lipid emulsion on bupivacaine plasma concentration in humans. Anaesthesia. 2012;67:600-5.

17. Presley JD, Chyka PA. Intravenous lipid emulsion to reverse acute drug toxicity in pediatric patients. Ann Pharmacother. 2013;47:735-43.

18. French D, Smollin C, Ruan W, Wong A, Drasner K, Wu AH. Partition constant and volume of distribution as predictors of clinical efficacy of lipid rescue for toxicological emergencies. Clin Toxicol (Phila) 2011;49:801-9.

19. Samuels TL, Willers JW, Uncles DR, Monteiro R, Halloran C, Dai H. In vitro suppression of drug-induced methaemoglobin formation by Intralipid $^{\circledR}$ in whole human blood: observations relevant to the 'lipid sink theory'. Anaesthesia. 2012;67: 23-32.

20. Kuo I, Akpa BS. Validity of the lipid sink as a mechanism for the reversal of local anesthetic systemic toxicity: a physiologically based pharmacokinetic model study. Anesthesiology. 2013;118: 1350-61. 
21. Killoran PV, Cattano D. From bedside to bench and back: perfecting lipid emulsion therapy for local anesthetic toxicity. Anesthesiology. 2011;115:1151-2.

22. Liu L, Xia Y, Chen Y, Wang Q, Shi T, Wang F, Small RH, $\mathrm{Xu} \mathrm{X}$. The comparative effects of lipid, epinephrine, and their combination in the reversal of bupivacaine-induced asystole in the isolated rat heart. Anesth Analg. 2012;114: 886-93.

23. Levine M, Brooks DE, Franken A, Graham R. Delayed-onset seizure and cardiac arrest after amitriptyline overdose, treated with intravenous lipid emulsion therapy. Pediatrics. 2012;130: e432-8.
24. Hendron D, Menagh G, Sandilands EA, Scullion D. Tricyclic antidepressant overdose in a toddler treated with intravenous lipid emulsion. Pediatrics. 2011;128:e1628-32.

25. Sirianni AJ, Osterhoudt KC, Calello DP, Muller AA, Waterhouse MR, Goodkin MB, Weinberg GL, Henretig FM. Use of lipid emulsion in the resuscitation of a patient with prolonged cardiovascular collapse after overdose of bupropion and lamotrigine. Ann Emerg Med. 2008;51:412-5.

26. Niiya T, Litonius E, Petaja L, Neuvonen PJ, Rosenberg PH. Intravenous lipid emulsion sequesters amiodarone in plasma and eliminates its hypotensive action in pigs. Ann Emerg Med. 2010;56:402-8 e2. 